\section{L'Archeologia dell'architettura in Italia nell'ultimo quinquennio (1997-2001)}

Gian Pietro Brogiolo

Università degli Studi di Padova

\begin{abstract}
Riassunto
Il contributo delinea un bilancio dell'Archeologia dell'architettura in Italia a partire dalla metà degli anni ' 90 del XX secolo, quando alcuni convegni e la neonata rivista "Archeologia dell'Architettura", misero a confronto le esperienze maturate in più centri di ricerca, che riguardavano non solo l'analisi stratigrafica delle murature il suo rapporto con il Restauro, ma anche allo studio delle tecniche costruttive, della mensiocronologia e dell'archeometria. Da questo punto di vista sono da segnalare da un lato le sperimentazioni nella costruzione delle sequenze degli equilibri statici, del degrado, degli intonaci e degli orizzontamenti lignei, dall'altro le proposte di metodologie di restauro fondate sull'analisi stratigrafica. Questo impegno ha però portato a trascurare l'obiettivo prioritario dell'archeologo: recuperare dalle sequenze di un edificio informazioni storiche, per le quali servono anzitutto corpora e censimenti esaustivi, e studi che pongano in relazione le architetture con l'organizzazione agraria, le trasformazioni dei paesaggi antropici, le trasformazioni economiche e sociali. Ed è su questo aspetto che converrà puntare nei prossimi anni, almeno da parte degli archeologi, senza per questo sminuire o vanificare il rapporto privilegiato che si è instaurato negli anni ' 90 con il Restauro Architettonico, con l'obiettivo comune di salvaguardare il patrimonio architettonico in una congiuntura nella quale sembra concluso un ciclo storico che aveva a cuore lo studio e la tutela del passato, attraverso il policentrismo culturale, la fervida circolazione delle idee, un saldo collegamento con la società civile.
\end{abstract}

Parole chiave: Archeologia dell'architettura, Italia, Restauro architettonico, Interpretazione storica.

\section{Abstract}

The author makes a summary on the situation of Building Archaeology in Italy from the 1990s onwards, when the creation of the journal "Archeologia dell'architettura" and several Congresses made it possible for different groups of investigators to share their strategies and experience, both regarding stratigraphic readings and their relationship to restoration and the study of buildings techniques, dimensional chronology and archaeometry. A key argument in this debate has been the application of this set of methods to architectural restoration, and experimentation on the static equilibrium sequence, degradation and wall coating as crucial instruments in a correct restoration process. This debate has given lesser focus to the main goal of the archaeologist: to obtain historical information from stratigraphic reconstructions, for which becomes in turn necessary to prepare repertoires and undertake extensive studies, relate archaeology to agrarian structures and landscape transformations as well as with social and economic structures. Archaeologists must work on this issue on the following years, without leaving aside the intimate relationship established with architectural restoration, to preserve the building patrimony in a context which should promote cultural multicentralism, the exchange of ideas and a recovery of civil society.
\end{abstract}

Key words: Building Archaeology, Italy, Architectural Restoration, Historical interpretation.
L'invito rivoltomi dagli organizzatori del Convegno a delineare un bilancio e riflettere sulle prospettive dell'Archeologia dell'Architettura in Italia mi ha riportato, dopo alcuni anni di distacco, ad un tema da me affrontato in più occasioni, sia per gli aspetti metodologici intrinseci alla disciplina (BROGIOLO, 1988a-b, 1997b) sia in rapporto al Restauro architettonico (BROGIOLO, 1993, 1995; Brogiolo et alii, 2000).

Per la prima parte del mio intervento, dedicata ad un bilancio, prenderò dunque le mosse dal mio ultimo contributo, scritto nel 1996, se non altro per confrontare le idee di allora con la situazione odierna.

Gli anni 1995-1996 furono importanti per lo sviluppo della nuova disciplina in Italia, così come lo era stato il 1987, quando al corso tenuto alla Summer School di Pontignano (Siena) ne vennero poste le basi teoriche e metodologiche (Francovich, Parenti, 1988).

Si tennero infatti quattro convegni, a Brescia, Milano, Trento e Genova, che raccolsero al tavolo della discussione archeologi e architetti. Risultato non secondario di quegli incontri fu l'avvio della Rivista, supplemento di Archeologia Medievale, che, per definire il variegato approccio archeologico agli edifici storici, si diede un titolo innovativo (Archeologia dell'Architettura) rispetto a quelli impiegati in precedenza: Archeologia stratigrafica degli alzati, Archeologia dell'edilizia storica, Rilievo critico dell'architettura.

L'ambizione era, in primo luogo, di riunire sotto un'etichetta comune esperienze maturate in più centri di ricerca (Siena, Genova, Venezia, Brescia e poi Roma, Milano etc.), rivolte non solo all'analisi stratigrafica delle murature (PARenti, 1985, 1988; Doglioni, Gabbiani 1985; Doglioni, 1997; Brogiolo, 1988a) e al suo rapporto con il Restauro, ma anche allo studio delle tecniche costruttive (Mannoni, 1994; Parenti, 1994; Cagnana, 2000), della mensiocronologia e dell'archeometria, per poi gettare dei ponti in direzione di altre discipline ormai consolidate, quali la storia dell'architettura, la geografia e l'etnografia degli insediamenti (alla Gambi e alla Marano), alla storia tout court (pensavo allora all'ottimo contributo di Etienne Hubert sull'edilizia bassomedievale romana).

Dal momento che l'avventura aveva avuto come partners, fin dalla seconda metà degli anni ' 80 , gli architetti restauratori, una parte essenziale del dibattito riguardò il rapporto con il Restauro.

Derivano da questo impegno anche alcune sperimentazioni di quegli anni, volte ad ampliare l'archeologia stratigrafica, tradizionalmente ristretta alle murature, ad una serie di sequenze (degli equilibri statici, del degrado, degli 
intonaci e degli orizzontamenti lignei) che hanno peculiare importanza nella costruzione di un corretto itinerario di restauro.

Vedevo peraltro anche $\mathrm{i}$ rischi insiti in un abbraccio troppo stretto da parte dei restauratori, che avrebbe potuto portare ad una devianza rispetto all'obiettivo prioritario dell'archeologo: recuperare dalle sequenze di un edificio dati, tracce, indizi storici, non dimenticando che la stratificazione comincia con gli alzati, ma prosegue nei depositi sepolti, senza soluzione di continuità storica, pur con una suddivisione artificiosa dovuta alle diverse specializzazioni e, in Italia, anche alle differenti competenze nella tutela.

Ribadivo anche la necessità di costruire atlanti delle tecniche costruttive, dei materiali, dei diversi tipi edilizi, strumento indispensabile per passare poi a studi più generali su singole classi di edifici, coniugando lo studio formale (stilistico), proprio della storia dell'architettura, con quello (storico) dell'evoluzione dei cicli produttivi in rapporto alla variabile specializzazione delle maestranze.

Temi che trovavano un campo privilegiato di verifica dall'età di transizione all'affermazione del Romanico, lungo periodo nel quale sono da correlare e spiegare su un piano storico il variare (etnico e culturale) delle classi egemoni in rapporto alla trasformazione delle architetture.

Altra componente fondamentale era l'impegno per la tutela: la ricerca vista come una premessa per una progettazione delle trasformazioni nel territorio. Una componente, forse utopica, ma che è ancora assai attiva nel seno dell'Archeologia Medievale, come confermano il Convegno di Milano del 1999 (De Marchi, Scudellari, Zavaglia, 2001) e la Summer School di Pontignano del 2000 (Atti in corso di stampa a cura di Andreina Ricci), dedicati ad Archeologia e Urbanistica (tema sul quale tornerò più avanti).

Queste erano dunque le idee che circolavano alla metà degli anni '90. Ma cosa è successo in seguito? Nella seconda metà degli anni '90, gli archeologi e gli architetti, che nel decennio precedente avevano sviluppato teoria e metodi nell'architettura stratigrafica, sono stati coinvolti in un prolungato dibattito sulle ricadute applicative nel campo del restauro architettonico. I convegni di Brescia (Della Torre, 1996), Milano (De Marchi, Mailland, Zavaglia, 1998), Genova (in Archeologia dell'architettura, II), Bressanone (AA. VV., 1996), i seminari di Padova (senza atti), Trento (CAvadA, Gentilini, 2000), Siena (senza atti), hanno consentito un'esauriente discussione, ma hanno da un lato messo in luce le posizioni apparentemente inconciliabili delle diverse scuole di Restauro, in particolare quelle di Venezia (Doglioni), Milano (Treccani), Genova (Mannoni), dall'altro hanno rivelato una fase della ricerca ancora assai vitale, anche se talora magmatica ed orientata in più direzioni.

Tentiamone un sintetico bilancio in sei punti, cominciando dal tema che è stato dominante nella seconda metà degli anni '90.

\section{ARCHEOLOGIA DELL'ARCHITETTURA E RESTAURO}

Sulla riflessione, teorica e metodologica, che concerne i rapporti tra Archeologia dell'architettura e Restauro, il contributo più rilevante, negli ultimi anni, è venuto dagli architetti.

In primo luogo, come critica delle "mentalità e pratiche dell'archeologia nell'intervento sul costruito": tra virgolette il sottotitolo di un volume di Gian Paolo Treccani (2000), che ha rimproverato agli archeologi una scarsa coscienza storicistica e, talora, una certa brutalità nell'intervento sul costruito, coniugata al semplicismo nel disfarsi delle cosiddette superfetazioni che celano il "tesoro" antico, da riportare in luce a scapito delle sequenze successive. Il mio parere (BrogIOLO, 1995), come quello di Doglioni (1997), rispetto a queste critiche, avanzate dal gruppo milanese fin dal convegno di Brescia del 1995, è che la conoscenza non si debba fermare di fronte ad un eventuale parziale sacrificio delle stratigrafie. Questa critica peraltro non scalfisce una valutazione positiva dell'analisi stratigrafica che però si deve fermare di fronte agli intonaci, anche recenti, che nascondo no le sequenze più antiche.

Più costruttiva mi pare la proposta di utilizzo (strumentale) dell'archeologia stratigrafica come guida al progetto di restauro, non a caso definito "stratigrafico", e come controllo delle trasformazioni indotte dagli interventi di restauro, temi trattati con una ricca esemplificazione nel volume di Doglioni del 1997 che riassume le esperienze avviate fin dalla metà degli anni ' 80 dal gruppo veneziano, al quale si può aggiungere un lavoro comune sul castello di S. Croce di Cervarese (Brogrolo et alii, 2000).

Il progetto stratigrafico, sul quale Doglioni ha riproposto alcuni temi in questo convegno attraverso esempi inediti, non pretende certo di imporre una linea progettuale unica e coercitiva. È una scelta che a mio avviso deve avere cittadinanza, senza suscitare preconcette levate di scudi, così come altre scelte progettuali possono risultare accettabili. La visione di una parete a palinsesto, che certo, più che altre filosofie del restauro, gratifica lo stratigrafo, può essere appagante anche per altri utenti dell'edificio 
restaurato, soprattutto se si accompagna ad un apparato didattico esplicativo che spiega la molteplicità di informazioni rintracciabili in quel paramento. Una scelta questa che trovo particolarmente efficace, ma raramente praticata, nei progetti che concernono monumenti o edifici destinati a musei, luoghi che per definizione dovrebbero essere un concentrato di significati storici.

Non sono state opposte obiezioni di principio sulla proposta, da me avanzata nel 1993 (Brogiolo, 1993) e sperimentata poi efficacemente da Doglioni, di servirsi dell'analisi stratigrafica, oltre che come strumento di conoscenza, anche per una verifica delle trasformazioni dello spessore storico di un edificio prodotte da un intervento di restauro.

Un ulteriore problema, sul quale si sono soffermate, in questi ultimi anni, sia la scuola veneziana sia quella milanese, concerne la gestione dei grandi numeri delle informazioni prodotte dall'analisi stratigrafica: la prima proponendo di trasferire la maggior parte dei dati stratigrafici sul disegno, la seconda elaborando sistemi di registrazioni e di analisi che coniugano il dato stratigrafico con le prescrizione di restauro (a tal proposito rimando all'intervento di Pertot in questo stesso convegno).

A ben vedere, nell'ampio dibattito che ha coinvolto le varie "scuole" di Restauro e di Archeologia dell'architettura non sono mancati taluni risvolti negativi. Ciascuna ha infatti sviluppato un proprio pensiero critico, più $o$ meno complesso, riconducibile con difficoltà ad una base teorica e metodologica comune.

La divisione, il particolarismo, il desiderio di sperimentare nuovi percorsi possono essere sì valutati come una ricchezza della disciplina, ma inducono altresì al rischio dell'incomprensione e dell'isolamento di chi pratica la stratigrafia rispetto al vasto mondo di chi restaura monumenti facendone tranquillamente a meno. Ad esempio nel manuale della Giusti (2000) non se ne fa alcun cenno e la "carta del rischio", di cui si dirà più avanti, prescinde dai metodi stratigrafici. Su un altro versante, nella presentazione dell'ultimo congresso italiano di Archeologia Cristiana, si rivendica la primogenitura dei cristianisti anche in questo campo. Tutto ciò è sintomatico della persistente debolezza della disciplina. Sarebbe perciò auspicabile, così come è avvenuto per lo scavo stratigrafico, trovare una convergenza almeno su alcuni principi comuni accettati da tutti.

\section{MATERIALI, TECNICHE COSTRUTTIVE, TIPI EDILIZI}

Un secondo tema sul quale si è lavorato con assiduità, a Siena come a Genova, è quello della conoscenza estensiva dei materiali, delle tecniche costruttive, come delle diverse tipologie edilizie (edifici rurali, opifici, case urbane, castelli ecc.).

In questo campo va segnalato l'agile manuale sulle tecniche costruttive pubblicato nel 2000 da Aurora Cagnana, allieva di Tiziano Mannoni, mentre siamo in attesa della prossima pubblicazione del nuovo manuale di Archeologia dell'architettura promesso da Roberto Parenti.

Temi più specifici, in un ambito regionale, sono stati affrontati da Tiziano Mannoni e Giovanna Bianchi, trattando della cronotipologia delle murature storiche in pietra, della mensiocronologia dei laterizi e dei segni di riconoscimento dei tagliapietra toscani.

Al di là di questi studi, rimarchevoli per l'approccio metodologico originale, permane il problema della costruzione di "corpora".

Non si tratta solo di reperire le risorse umane e finanziarie, ma anche di convincere lo Stato italiano, che si è riservato la tutela, che le schedature indiscriminate ed occasionali realizzate sinora nell'ambito del Catalogo Nazionale dei Beni Culturali non hanno prodotto alcun rilevante risultato scientifico e nemmeno sono servite a salvaguardare il nostro patrimonio. Anche l'ultima invenzione, scaturita dalla constatazione di questi fallimenti e denominata "carta del rischio", si muove nella vecchia logica dell'intervento casuale. Il progetto è stato varato dal ministero per i Beni e le Attività Culturali in collaborazione con alcune regioni (SESSA, 2000). Per la documentazione delle architetture incluse nella carta vengono utilizzate le schede descrittive dell'Istituto Centrale per il catalogo e la documentazione, che prevedono la registrazione dello spessore storico in una voce secondaria denominata "preesistenze".

Anche la scelta degli edifici da censire appare discutibile. Sono stati infatti inseriti, in prima battuta, quelli segnalati nelle Guide Rosse del Touring, considerati a ragione o a torto di peculiare pregio storico-artistico, a scapito del palinsesto architettonico complessivo presente in ciascuna area regionale.

D'altra parte, la "carta del rischio" si adatta perfettamente alla politica riduttiva del Ministero dei Beni Culturali che, al di là delle enunciazioni di principio su una salvaguardia a $360^{\circ}$ contenute negli ultimi decreti legislativi, non riesce ad impedire le distruzioni prodotte da uno sviluppo urbanistico in larga misura incontrollato.

Avrebbe avuto maggior senso articolare il censimento per categorie o classi di edifici, come è stato fatto in Toscana dall'Università di Siena che in pochi anni ha sche- 
dato oltre 2500 castelli attraverso strumenti sistematici quali la foto area e le fonti scritte, per passare poi alla documentazione a campione sul terreno.

Censimenti esaustivi divengono in tal modo strumenti di base per avviare poi studi su specifici temi di grande rilevanza storica, come quelli che Riccardo Francovich e Giovanna Bianchi hanno presentato proprio a questo convegno.

Proprio l'esempio senese dimostra che quando si riesce a reperire un modesto finanziamento, di gran lunga inferiore ai fondi investiti da alcune regioni nella "carta del rischio", non mancano le risorse umane per iniziative di grande portata.

Concentrarsi su un settore significa lasciar perdere altre ricerche ugualmente interessanti, ma operare episodicamente a $360^{\circ}$ si traduce troppo spesso in una dispersione delle risorse, senza che di quel tema si costruiscano i fondamenti.

Di fronte alle scelte degli organi ministeriali italiani che vanno in tutt'altra direzione, non resta che ricorrere a finanziamenti locali o a fondi europei, che consentano ricerche in contesti storico culturali per molti periodi storici di fatto unitari.

Qualche timido segnale di collaborazione internazionale si comincia ad avvertire, ad esempio nell'avvio di un progetto di censimento delle chiese europee dalle origini al X secolo, opera monumentale che dovrebbe darci un quadro delle manifestazioni architettoniche più diffuse $\mathrm{e}$ meglio conservate dell'età altomedievale.

$\mathrm{Ma}$ anche altri atlanti, nell'ambito dell'edilizia fortificatoria e residenziale, sarebbero necessari prima che le trasformazioni urbanistiche in atto cancellino questa preziosa fonte materiale.

\section{ARCHEOLOGIA E STORIA DELL'ARTE}

Quirós Castillo, riprendendo criticamente negli atti del secondo congresso italiano di archeologia medievale un mio contributo degli anni ' 80 , ha ribadito la totale impermeabilità tra archeologi dell'architettura e storici dell'arte (Pierotti, Quirós Castillo, 2000).

Ritengo tuttavia che l'assenza di questi ultimi dal dibattito suscitato da archeologi ed architetti restauratori, non dipenda, come sostiene Quirós, dal fatto che solo da poco gli archeologi hanno cominciato ad occuparsi di monumenti di prestigio, ma derivi piuttosto, almeno da parte di alcuni, da una estraneità preconcetta nei confronti di una disciplina ancor giovane e priva di blasoni. Si legga al riguardo la presentazione al IX congresso nazio- nale di Archeologia Cristiana nella quale l'autore addebita agli archeologi dell'architettura di "passare completamente sotto silenzio quanto è stato fatto al riguardo prima e dopo gli anni '70 dagli archeologi cristiani, in particolare dal Krautheimer fin dagli anni '30", in un clima "di affermazione e di autocelebrazione di una disciplina di recente introduzione rispetto all'Archeologia Cristiana, che ha più di 400 anni alle spalle" (RUSSO in AA.VV., 2001).

Una maggior umiltà da entrambe le parti non guasterebbe. Gli archeologi, talora trascinati dall'entusiasmo di intraprendere nuovi percorsi epistemologici, dimenticano i risultati di discipline, magari meno attente al dettaglio e agli aspetti materiali, ma pur sempre in grado di produrre sintesi nelle quali essi non si sono ancora cimentati.

"I nostri maestri - scriveva il compianto archeologo ticinese Pier Angelo Donati (1999: 199) -, avevano una visione più completa dell'insieme, forse incompleta nel dettaglio tecnico-metodologico, ma tale da rendere più accessibile la materia". Gli stratigrafi dimenticano inoltre che ancora oggi come in passato, sono state realizzate sequenze corrette di architetture, pur senza la formalizzazione e un percorso logico derivati dai metodi harrisiani (si vedano ad esempio i contributi della scuola tedesca di restauro).

Sull'altro versante, alcuni storici dell'arte mi paiono fin troppo precipitosi nel liquidare come periferico e sussidiario tutto ciò che non rientra nei canoni tradizionali della loro disciplina. I contributi di Fabio Gabbrielli sulle aperture della facciata del palazzo pubblico di Siena (1996) e sulle arcate della chiesa monastica di San Galgano (1998, 2000) suggeriscono invece una possibile convergenza. La stereotomia e la cronotipologia applicate agli elementi architettonici richiedono infatti un parallelo approccio storico artistico ed archeologico in grado di assicurare un risultato storico convergente che dimostra come nella costruzione di un medesimo monumento siano potuti intervenire gruppi di artigiani con diverse capacità ed esperienze tecniche.

È dunque auspicabile che si attenuino le barriere che ciascuna disciplina ha eretto per difendere la propria genuinità dalle interferenze. La ricerca scientifica non si sviluppa costruendo fortini, ma dialogando. Sulle architetture, come in ogni campo della ricerca, non possono estendersi riserve privilegiate di qualsivoglia disciplina. Lo specialista appagato del metodo e dell'epistemologia della sua disciplina, se non sa dialogare con gli altri studiosi, si condanna ad una lettura parziale e ad una interpretazione altrettanto circoscritta della realtà storica. 


\section{ARCHEOLOGIA DELL'ARCHITETTURA E INTERPRETAZIONE STORICA}

Il fine ultimo di chi fa archeologia, al quale non si sottraggono quanti operano nell'architettura, è il fare storia, ossia il ricostruire, attraverso una delle testimonianze ancora oggi più diffuse, una serie di profili storici del passato, dai quadri economici all'organizzazione delle produzioni, dalla cultura all'ideologia di specifici gruppi sociali.

Muovendosi su questa linea alcuni giovani ricercatori, a partire dalla metà degli anni novanta hanno cercato di ricostruire i cicli edilizi e le trasformazioni delle tecniche costruttive in rapporto alla committenza e alle maestranze. Giovanna Bianchi (1997) ha proposto l'introduzione di concetti, ripresi dall'antropologia culturale, quali "sapere tecnico" inteso come conoscenze e capacità artigianali di un gruppo umano e “interazione interno-esterno". Concetti valutati nel rapporto tra maestranze itineranti e maestranze locali, la cui attività costruttiva nei castelli toscani interagisce nella formazione di specifiche tecniche costruttive. La fine dell'architettura residenziale in legno, predominante nell'alto medioevo fino al X secolo, e l'affermazione di quella in muratura tra quel secolo e il successivo, sono state ricondotte ad una serie di relazioni dicotomiche tra città e campagna, tra architetture religiose, con poche eccezioni da sempre realizzate in muratura, e architetture di potere civile che persistono in legno fino a tutto il X secolo.

Si è proposta una prospettiva storica anche per l'analisi mensiocronologica applicata ai laterizi tardomedievali e postmedievali della Toscana nord occidentale. Verifica peraltro limitata, in questa regione, dalla frammentazione dei mercati. A causa della mancata integrazione tra città e campagna la durata degli impianti produttivi fissi si conclude nell'ambito di due o tre generazioni e prevalenti sono dunque in quel territorio le fornaci occasionali, attivate per specifici monumenti. (Quirós CASTILLO, 1997)

\section{ARCHEOLOGIA DELL'ARCHITETTURA E URBANISTICA}

Un tema che ha trovato accoglienza nell'archeologia medievale fin dagli anni '70 è stato quello del possibile contributo alla pianificazione urbanistica da parte dell'archeologia. Anche l'archeologia dell'architettura, nella prospettiva "militante", non si è voluta sottrarre a questo impegno tentando di coinvolgere nell'impresa geografi ed architetti.

Tuttavia, nell'applicazione alla pianificazione urbanistica dei metodi, e soprattutto dei risultati, delle analisi estensive dell'Archeologia dell'architettura, gli archeologi si sono trovati prevalentemente soli.
Le proposte, avanzate di volta in volta da Parenti (1992) e dal gruppo che fa capo a Francovich, non hanno trovato particolare udienza nel mondo degli urbanisti. Ho l'impressione che da parte degli architetti il rapporto tra Archeologia e Urbanistica venga inteso esclusivamente come gestione del patrimonio archeologico all'interno della pianificazione territoriale. Su questo tema il dibattito è sempre stato assai vivo: dallo scontro sugli scavi dei Fori Imperiali, a quelli del Policlinico di Napoli, ai parchi archeologici suburbani di Roma. Più recentemente se ne è discusso nel convegno che ha fatto il punto sull'Archeologia urbana, dalla valutazione alla gestione dei depositi archeologici delle città, nel convegno di Milano del 1999 su "Lo spessore storico in urbanistica" (DE Marchi, Scudellari, Zavaglia, 2001) e, nel febbraio 2000, al seminario di Siena, organizzato da Andreina Ricci, alla quale si deve anche un interessante intervento sulla rivista "Archeologia Medievale" del 1999.

In queste discussioni è stata ribadita con chiarezza l'opportunità delle analisi stratigrafiche estensive nella pianificazione urbanistica. Per quanto riguarda lo studio dei centri storici, che il metodo sia applicabile e che abbia costi compatibili con quelli di uno strumento urbanistico è stato dimostrato fin dalla fine degli anni ' 70 - inizi anni '80 in Lombardia nello studio dei centri urbani di Erbanno e Gorzone (1979), di Pescarzo (1984-1985). Sulla scia di questi lavori, che non hanno avuto a suo tempo adeguata pubblicazione, si colloca anche la recente e ottima ricerca su Cividate Camuno (BIANCHI, MaCARIO, ZonCA, 1999). D'altra parte, l'applicazione del GIS, sperimentata a Campiglia (BIANCHI, NARDINI, 2000), non può che contribuire ad una fruizione più rapida dei dati raccolti.

\section{ARCHEOLOGIA DELL'ARCHITETTURA E INFORMATICA}

Indispensabile per la gestione della grande massa di informazioni raccolte nell'analisi di un edificio complesso, di più edifici o di un intero centro storico, appare lo strumento informatico, in particolare il GIS. Le esemplificazioni presentate dal gruppo senese al II Congresso di Archeologia medievale per Campiglia Marittima (Bianchi, Nardini, 2000) e da Tiziano Mannoni per il centro storico di Genova (MANNONI, 2000; VECCHIATTINI, 2001) hanno tolto ogni dubbio sulla sua utilità, se mai ne restavano. Nelle architetture appare però indispensabile passare da un GIS bidimensionale, nel cui ambito rimangono gli esempi che ho citato, ad uno tridimensionale in grado di collegare tra loro le informazioni stratigrafiche, materiche, formali che in un edificio si distribuiscono per l'appunto nelle tre dimensioni. Quanto ci è stato mostrato 
dagli amici spagnoli proprio qui a Vitoria sull'applicazione di un GIS tridimensionale nel progetto della cattedrale mi pare vada proprio nella direzione auspicata.

\section{CONCLUSIONI E PROSPETTIVE}

Nonostante la preoccupazione di fondo sulla tenuta della disciplina, mi pare che il bilancio di questo quinquennio sia in una certa misura positivo.

L'archeologia stratigrafica viene accettata in molti cantieri come strumento funzionale alla conoscenza e come guida indispensabile ad un progetto critico, anche se permane, occorre sottolinearlo, una diffusione regionale di queste pratiche. Molti centri accademici e gli organi centrali dello stesso Ministero per i Beni Culturali ne restano prevalentemente immuni.

Si ha inoltre l'impressione che, nella maggior parte dei casi nei quali l'archeologia stratigrafica viene coinvolta in un progetto di restauro, non esca da una posizione ancillare, non diventi un effettivo ed autonomo momento di conoscenza approfondita e pluridirezionale, così come i lavori sperimentali che ho ricordato hanno saputo definire.

Lavori che cercando di colmare il distacco dall'Archeologia medievale, intesa come studio unitario delle stratigrafie sepolte e di quelle in alzato e le divaricazioni tra fonti scritte, lettura storico artistica e dato materiale, hanno indicato le strade attraverso le quali raggiungere l'obiettivo prioritario di una qualsiasi ricerca archeologica: produrre una rilettura storica. È questa una prospettiva che da sola giustifica un futuro all'Archeologia dell'architettura, che deve farsi Archeologia delle architetture, ricostruendo la storia degli edifici come parte della più generale storia delle società del passato.

Ma per raggiungere questo obiettivo è indispensabile superare la strettoia dei "corpora": di chiese, castelli, edifici residenziali ecc., strumento di base per avviare ricerche più approfondite su temi generali, quali ad esempio su architetture militari bizantine ed edilizia religiosa di VI secolo in Italia e nel Mediterraneo, su maestranze itineranti e committenza presso le corti longobarde, su centri e modalità di sviluppo del romanico. Ovvero, nella prospettiva di una ricostruzione storica "totale", per indagare in limitate aree campione l'evoluzione dell'insieme delle architetture in rapporto al variare dell'organizzazione agraria e alle trasformazioni dei paesaggi antropici, per comprendere ad esempio le interazioni tra architetture e organizzazione signorile o altre forme di gestione delle campagne.

Questi obiettivi storici non devono però sminuire o vanificare il rapporto privilegiato che si è instaurato negli anni '90 con il Restauro Architettonico; il confronto deve trovare una rinsaldata convergenza di interessi nell'impegno civile per la tutela del patrimonio architettonico in una fase cruciale nella quale sembra attenuarsi per tutti noi, archeologi e architetti militanti, un ciclo storico basato sul policentrismo, sulla fervida circolazione delle idee, sul collegamento con la società civile (BroGiolo, 1997a; GeLICHI, 2001). Un raccordo è tanto più utile nel momento in cui la nuova legislazione italiana da un lato amplia a dismisura il concetto di bene culturale fino a comprendere "le cose immobili e mobili che presentano interesse artistico, storico, archeologico o demo-etno-antropologico", quali le fotografie e i mezzi di trasporto con più di 75 anni, ma dall'altro lascia il tessuto edilizio storico alla gestione urbanistica delle Regioni. Alcune delle quali ne hanno decretato la condanna, ammettendo demolizioni e ricostruzioni generalizzate (si veda ad esempio quanto previsto dalla legge regionale 23 della Regione Lombardia).

Di fronte al fallimento della programmazione urbanistica si sono sostituiti interventi occasionali e puntifor$\mathrm{mi}$, in un quadro legislativo che, tra condoni e abolizione di controlli, lascia spazio all'arbitrio e agli interessi particolari.

A pochi mesi dal convegno di Vitoria, mentre sto rivedendo il testo letto in quella sede, il dibattito in Italia si è rianimato a seguito della presentazione di una nuova legge, che prevede la possibilità di cedere il patrimonio dello Stato, compresi i Beni Culturali, a due Società per azioni, dunque di diritto privato, allo scopo di valorizzare, gestire ed eventualmente alienare il patrimonio pubblico, compresi monumenti, parchi naturali ed archeologici, musei. "Il patrimonio culturale italiano - scrive Salvatore Settis su "La Repubblica" del 29 giugno 2002 - non è mai stato tanto minacciato quanto oggi, nemmeno durante guerre e invasioni: perché oggi la minaccia viene dall'interno dello Stato". Il rischio, rimarcano le associazioni ambientalistiche e gli addetti ai lavori, è in una valutazione esclusivamente economicistica dei Beni Culturali, il che significa tornare, come in passato, a privilegiare le grandi opere, a scapito della conservazione di ciò che, pur avendo un grande rilievo storico, ha scarso valore artistico e dunque anche venale, come è il caso della maggior parte delle architetture del passato.

In conclusione l'Archeologia dell'architettura in Italia sembra collocarsi, a medio termine, tra due prospettive: da un lato l'opportunità di perseguire obiettivi di conoscenza storica ricercando sinergie e collaborazione con le altre discipline che si occupano del costruito; dall'altro la necessità di mantenere vivo il rapporto con chi opera 
sulle trasformazioni delle architetture per una assunzione di responsabilità comune nella salvaguardia.

Senza un impegno rinnovato a scala europea, oltre al patrimonio archeologico ed architettonico rischiano di essere travolti anche gli ideali che hanno animato l'Archeologia medievale italiana, fin dalla sua rifondazione 30 anni orsono. Non dimentichiamo che, dopo una promettente fase di incubazione nella seconda metà del XIX secolo che aveva visto architetti ed archeologi collaborare, venne insegnata da Monneret de Villard al Politecnico di Milano fin dagli inizi del XX secolo (Augenti, 2001), ma non ha poi trovato spazio nell'insegnamento universitario fino agli anni '70 del XX secolo.

La totale indifferenza dei partiti politici, della maggior parte dei media e dunque dell'opinione pubblica al dibattito sui beni culturali italiani di questi ultimi anni, ci avverte che il rischio è generale e non riguarda solo le nostre discipline, sviluppatesi anche nei paesi latini, negli anni '70-80 del XX secolo, sulla scia della rivalutazione della cultura materiale promossa dal neopositivismo e dal neomarxismo. Le società europee vanno cambiando sulla spinta di una crisi di cui stentiamo a definire le coordinate e di nuove ideologie che avanzano cercando altrove $i$ propri valori. Lo studio e la salvaguardia del passato appaiono ora meno importanti che cinquant'anni orsono. Le tradizioni hanno perso la loro capacità di coinvolgere la popolazione, forse perché nelle società multiculturali e dominate dalla telematica non costituiscono più il riferimento di un comune sentire (MULGAN, 1998).

\section{Bibliografía}

AA. VV., 1996, Dal sito archeologico all'archeologia del costruito. Conoscenza, Progetto e Conservazione, Padova.

AA. VV., 2001, Edificio battesimale in Italia. Aspetti e problemi, Atti dell'VIII Congresso Nazionale di Archeologia Cristiana, (1998).

Augenti A., 2001, Per una storia dell'archeologia medievale italiana: Ugo Monneret de Villard, Archeologia Medievale, XXVIII, pp. 7-24.

Bianchi A., MACARIO F., ZONCA A., 1999, Civethate, l'abitato e il territorio di Cividate Camuno in età medioevale, Breno.

BIANCHI G., 1996, Trasmissione dei saperi tecnici e analisi dei procedimenti costruttivi di età medievale, Archeologia dell'A rchitettura, I, pp. 53-64.

BiANCHI G., NARDINI A., 2000, Archeologia dell'architettura di un centro storico. Proposta per un'elaborazione informatica dei dati su piattaforma G.I.S. bidimensionale, Atti del II Congresso Nazionale di Archeologia Medievale, Brescia, pp. 381-388.

Brogiolo G. P., 1988a, Campionatura e obiettivi nell'analisi stratigrafica degli elevati, in R. Francovich, R. PARenti (a cura di), Archeologia e restauro dei monumenti. I ciclo di lezioni sulla ricerca applicata in archeologia, (Pontignano 1987), Firenze, pp. 335-346.

Brogiolo G. P., 1988b, L'analisi stratigrafica: un metodo per la lettura delle modificazioni architettoniche, Abacus, n. 14 (giugno-luglio 1988), pp.12-15.

Brogiolo G. P., 1993, Appunti su analisi stratigrafica e restauro, in M. UbOLDI (a cura di), Carta archeologica della Lombardia, III, Modena, pp. 103108.
Brogiolo G. P., 1995, Arquelogía estratigráfica y restauración, Informes de la construcción, 46, n. 435 (gen.-feb. 1995), pp. 31-36.

Brogiolo G. P., 1996, Prospettive per l'Archeologia dell'Architettura, Archeologia dell'Architettura, I, pp. 11-15.

BROGIOLO G. P., 1997a, Archeologia e Istituzioni: statalismo o policentrismo?, Archeologia Medievale, XXIV, pp. 7-30.

Brogiolo G. P., 1997b, Dall'analisi stratigrafica degli elevati all'Archeologia dell'Architettura, Archeologia dell'Architettura, II, pp. 181184.

Brogiolo G. P., 1998, Archeologia dell'Urbanistica: tra ricerca e tutela, in Cavada E., Gentilini G. (a cura di) 2000, Archeologia dei Centri Storici. Analisi conoscenza e conservazione, Atti seminario promosso dall'Associazione Ricerche Fortificazioni Altomedievali, (Trento), pp. 9-14.

Brogiolo G. P., Franceschi S., Lazzari A., 2000, Valutazioni e riflessioni sugli esiti del restauro compiuto nel castello di San Martino di Cervarese S. Croce (PD), Archeologia dell'Architettura, IV, pp. 247-258.

Cagnana A., 2000, Archeologia dei materiali da costruzione, Mantova.

Cavada E., Gentilini G., (a cura di) 2000, Archeologia dei Centri Storici. Analisi conoscenza e conservazione, Atti del seminario promosso dall'Associazione Ricerche Fortificazioni Altomedievali, (Trento 1998).

Della TORRE S., (a cura di) 1996, Storia delle tecniche murarie e tutela del costruito. Esperienze e questioni di metodo, Brescia, Milano.

De Marchi M., Mailland F., Zavaglia A., (a cura di) 1998, Lo spessore storico in architettura tra conservazione, restauro, distruzione, (Milano 1995), Milano, pp. 65-73

De Marchi M., Scudellari M., Zavaglia A., (a cura di) 2001, Lo spessore storico in urbanistica, (Milano 1999), Mantova.

DOGLIONI F., GABBIANI B., 1985, Metodologia per la conoscenza analitica del manufatto edilizio per il controllo tecnico-culturale dell'intervento di restauro, Venezia.

Doglioni F. (a cura di) 1987 , Ambienti di dimore medievali a Verona, Verona.

DOGLIONI F., 1988, La ricerca sulle strutture edilizie tra archeologia stratigrafica e restauro architettonico, in FrANCOVICH R., PARENTI R. (a cura di), Archeologia e Restauro dei monumenti. I ciclo di lezioni sulla ricerca applicata in archeologia. Certosa di Pontignano (Siena 28 settembre-10 ottobre 1987), Firenze, pp. 223-246.

DOGLIONI F., 1997, Stratigrafia e restauro. Tra conoscenza e conservazione dell'architettura, Trieste.

DONATI P., 1999, Sumirago. La chiesa di Santa Maria, in IDEM, Archeologia e monumenti del Ticino, Bellinzona, pp. 197-203.

FRANCOVICH R., PARENTI R. (a cura di), 1988, Archeologia e Restauro dei monumenti. I ciclo di lezioni sulla ricerca applicata in archeologia. Certosa di Pontignano (Siena) 28 settembre-10 ottobre 1987, Firenze.

GABBRIELLI F., 1996, La 'cronotipologia relativa' come metodo di analisi degli elevati: la facciata del Palazzo Pubblico di Siena, Archeologia dell'A rchitettura, I, pp. 15-44.

GABBRIELLI F., 1998, La chiesa dell'abbazia di S. Galgano. I. Stereotomia degli archi e fasi costruttive, Archeologia dell'Architettura, III, pp. 15-44.

GABBRIELLI F., 2000, La chiesa dell'abbazia di San Galgano. II. Stereotomia degli archi e delle maestranze, Archeologia dell'Architettura, V, pp. 25-62.

GELICHI S., (a cura di) 2001, Dalla carta del rischio archeologico di Cesena alla tutela preventiva urbana in Europa, Firenze, pp.15-17.

GiUsti M.A., (a cura di) 2000, Temi di restauro, Torino.

MANNONI T., 1994, Caratteri costruttivi dell'edilizia storica, 1994

MANNONi T., 2000, Dall'Archeologia globale del territorio alla Carta del patrimonio archeologico, architettonico e storico-ambientale, in CAVADA E., Gentilini G. (a cura di), Archeologia dei Centri Storici. Analisi conoscenza e conservazione, Atti seminario promosso dall'Associazione Ricerche Fortificazioni Altomedievali, (Trento 1998), pp. 21-27.

Mulgan G., 1998, Connexity: how to live in a connected world, Boston.

PARENTI R., 1985, La lettura stratigrafica delle murature in contesti archeologici e di restauro architettonico, Restauro e Città, I, n. 2, pp. 55-68.

PARENTI R., 1988, Le tecniche di documentazione per una lettura stratigrafica dell'elevato, in Francovich R., PARenti R. (a cura di), Archeologia e Restauro dei monumenti. I ciclo di lezioni sulla ricerca applicata in archeologia. Certosa di Pontignano (Siena) 28 settembre-10 ottobre 1987, Firenze, pp. 249-279. 
PARENTI R., 1992, Fonti materiali e lettura stratigrafica di un centro urbano: i risultati di una sperimentazione "non tradizionale", Archeologia Medievale, XIX, pp. 7-62.

PARENTI R., 1994, I materiali da costruzione, le tecniche di lavorazione e gli attrezzi, in G. P. Brogiolo (a cura di), Edilizia residenziale tra V e VIII secolo, Atti $4^{\circ}$ seminario sul tardoantico e l'altomedioevo in Italia centrosettentrionale, Monte Barro 2-4 settembre 1993, Mantova, pp. 25-37.

Paroli L., Delogu P., (a cura di) 1993, La storia economica di Roma nell'alto medioevo alla luce dei recenti scavi archeologici, Firenze.

Pierotti P., Quirós Castillo J. A., 2000, Archeologia dell'architettura e storia dell'architettura: due discipline a confronto, in Atti del II Congresso Nazionale di Archeologia Medievale, Brescia, pp. 377-380.

Quirós CASTILlO J.A., 1997, La mensiocronologia dei laterizi della Toscana: problematiche e prospettive di ricerca, Archeologia dell'Architettura, II, pp. 159-165.

RiCCI A., 1999, Luoghi estremi della città. Il progetto archeologico tra "memoria" e "uso pubblico della storia", Archeologia Medievale, XXVI, pp. 21 42.

SESSA V. M., 2000, La Carta del Rischio del patrimonio culturale: l'esperienza della Lombardia, Aedon, n.3.

TrecCANi G. P., 2000, Archeologie, restauro, conservazione. Mentalità e pratiche dell'archeologia nell'intervento del costruito, Milano.

VECCHIATTINI R., 2001, La mappatura culturale della città vecchia di Genova: un metodo per una lettura nuova della città, in M. DE MARCHI., M. SCUDellari, A. ZaVAGLia, (a cura di), Lo spessore storico in urbanistica, (Milano 1999), Mantova, pp. 129-142. 